\title{
EVALUATIONS OF THREE-DIMENSIONAL BUILDING MODEL RECONSTRUCTION FROM LIDAR POINT CLOUDS AND SINGLE-VIEW PERSPECTIVE IMAGERY
}

\author{
F. Tsai ${ }^{*}$ and H. Chang ${ }^{b}$ \\ ${ }^{\mathrm{a}}$ Center for Space and Remote Sensing Research \\ ${ }^{\mathrm{b}}$ Department of Civil Engineering \\ National Central University, Zhong-li, Taoyuan 320 Taiwan \\ ftsai@csrsr.ncu.edu.tw; 1984chang@gmail.com
}

KEY WORDS: 3D Building Model Reconstruction, LiDAR, Single View Reconstruction, Image-based Reconstruction, Close-range Photogrammetry

\begin{abstract}
:
This paper briefly presents two approaches for effective three-dimensional (3D) building model reconstruction from terrestrial laser scanning (TLS) data and single perspective view imagery and assesses their applicability to the reconstruction of 3D models of landmark or historical buildings. The collected LiDAR point clouds are registered based on conjugate points identified using a seven-parameter transformation system. Three dimensional models are generated using plan and surface fitting algorithms. The proposed single-view reconstruction (SVR) method is based on vanishing points and single-view metrology. More detailed models can also be generated according to semantic analysis of the façade images. Experimental results presented in this paper demonstrate that both TLS and SVR approaches can successfully produce accurate and detailed 3D building models from LiDAR point clouds or different types of single-view perspective images.
\end{abstract}

\section{INTRODUCTION}

Terrestrial laser scanning (TLS) or ground-based LiDAR (Light Detection And Ranging) and image-based measurement and reconstruction are two popular approaches for measuring and generating three-dimensional (3D) models of cultural heritage objects and sites. Both approaches have their advantages and disadvantages. The data acquired with terrestrial laser scanners are dense point clouds with accurate 3D coordinates, which can be used to reconstruct very detailed models of the targets. However, LiDAR point clouds are discrete data; they do not preserve the topology among returned point signals. Therefore, the processing of feature extraction and model reconstruction from LiDAR point clouds are complicated and relatively labor intensive and time consuming Pu and Vosselman (2009b,a). In addition, there are usually occlusions in the TLS data sets, especially the roof facets of buildings, because of the limitation in view angles of TLS sensors. On the other hand, image-based approaches have other advantages in comparison with in situ surveying and measurement. For example, it does not require expensive and heavy equipments and the cost is usually low. In addition to the geometric measurement and model reconstruction, image-based approaches can also produce accurate façades and surface textures to provide a more realistic look and feel of the generated models and scenes. Moreover, it is possible to restore the original models of historical objects and scenes even the targets are already destroyed or damaged, as long as there are still records comprising images of the buildings or site scenes of interest.

Conventional photogrammetric or vision-based methods require multiple images of the target with different view angles to undertake the reconstruction of 3D models. This sometimes can also be time consuming and the data might be difficult to acquire, especially for historical artifacts and buildings. Among the algorithms based on vision metrology, single view reconstruction (SVR) can reconstruct the 3D model of the visible parts of an object from a single image according to the perspective metrology (Chang and Tsai, 2009; Criminisi et al., 2000; Wang et al., 2005). It has a great potential for applications related to the digital 3D documentation and archiving of cultural heritage, especially for existing or historical landmark buildings.

This study explored both the TLS and SVR approaches for the generation of detailed 3D models of landmark buildings. The objective is two-folded. The first is to assess their applicability to digital documentation and archiving of cultural heritage objects. The second is to establish effective and systematic procedures for applying the technologies to reconstruct 3D models of culture heritage objects.

\section{TLS-BASED BUILDING MODELING}

Ground-based laser scanning has been demonstrated to be an effective approach for three-dimensional building model reconstruction, especially for detailed model reconstruction ( $\mathrm{Pu}$ and Vosselman, 2009b,a). For the TLS approach utilized in this study, LiDAR points collected from multiple scanning stations and, if necessary, combined with airborne laser scanning (ALS) data (to compensate the occlusions in roof structures, in particular) are registered using a seven-parameter transformation system of identified conjugate points to form a complete point cloud set of the target. As described in Eq. 1, the seven parameters include a scale factor, $S$, three offsets, $T_{X}, T_{Y}$, and $T_{Z}$, and three rotation angles $(\omega, \phi$, and $\kappa)$ which constitute the rotation matrices $\left(m_{11} \sim m_{33}\right)$.

$$
\left[\begin{array}{l}
X^{\prime} \\
Y^{\prime} \\
Z^{\prime}
\end{array}\right]=S\left[\begin{array}{lll}
m_{11} & m_{12} & m_{13} \\
m_{21} & m_{22} & m_{23} \\
m_{31} & m_{32} & m_{33}
\end{array}\right]\left[\begin{array}{c}
X \\
Y \\
Z
\end{array}\right]+\left[\begin{array}{c}
T_{X} \\
T_{Y} \\
T_{Z}
\end{array}\right]
$$




$$
\begin{aligned}
& m_{11}=\cos \phi \sin \kappa \\
& m_{12}=\sin \omega \sin \phi \cos \kappa+\cos \omega \sin \kappa \\
& m_{13}=-\cos \omega \sin \phi \cos \kappa+\sin \omega \sin \kappa \\
& m_{21}=\cos \phi \sin \kappa \\
& m_{22}=-\sin \omega \sin \phi \sin \kappa+\cos \omega \cos \kappa \\
& m_{23}=\cos \omega \sin \phi \sin \kappa+\sin \omega \cos \kappa \\
& m_{31}=\sin \phi \\
& m_{32}=-\sin \omega \cos \phi \\
& m_{33}=\cos \omega \cos \phi
\end{aligned}
$$

The point data are then partitioned with a surface growing algorithm, consisting of five steps: (1) seed decision, (2) seed plane generation, (3) plane surface points growing, (4) plane surface points merging, and (5) curve surface points generation. Finally, planar and curved surfaces are reconstructed using least-squares approximation and a semi-automatic curved surface fitting procedure, respectively. The Random Sample Consensus (RANSAC) algorithm Fischler and Bolles (1981) is utilized during the plan fitting process. RANSAC is a resampling technique to estimate model parameters and is designed to deal with data sets containing a large portion of outliers. A typical RANSAC-based algorithm for extracting a planar feature from a LiDAR point cloud is outlined in Algorithm 1. However, two types of curved surfaces are recognized and semi-automatically fitted in this study. The two curved surface types are roof and cylindrical structures. The type is determined by examining the vertical distribution of the points as shown in Fig. 1, where the blue points are the LiDAR points and the green dots are the gravity centers. After determining the type, parametric models of cylindrical surfaces are generated based on the radius and height measured from the partitioned point clouds, whereas the roof surfaces are approximated using rule surfaces constructed from outline curves.

\footnotetext{
Algorithm 1 RANSC for plan fitting from LiDAR point clouds

1. Randomly select 3 points.

2. Construct the plane model (solve the parameters of the plane equation).

3. Calculate the distance of a point to the plane, $d_{i}$, for all points.
}

4. Find inliers (points whose $d_{i}$ is less than a predefined threshold).

5. If the portion of the inliers over the total number of points is greater than a predefined threshold, reconstruct the plane based on all the identified inliers and terminate.

6. Otherwise, repeat steps 1 through 5 until reaching the maximum number of iteration, $\mathrm{N}$.

\section{SVR-BASED BUILDING MODELING}

For the SVR-based reconstruction of detailed 3D building models from single perspective view images, the developed method is modified from Chang and Tsai (2009), which is based on vanishing points. The procedure begins with edge detection and filtering processing of photographs or paintings with fine perspective geometry. Three mutually orthogonal vanishing points are then calculated automatically using a double Hough transform scheme applied to the identified line clusters. Feature points are extracted using a corner point filter with geometry constrains, and their corresponding ground points are predicted according to the vanishing points. The three-dimensional coordinates of feature points are also computed based on the vanishing points using singleview metrology. A height equalization algorithm and a vanishing
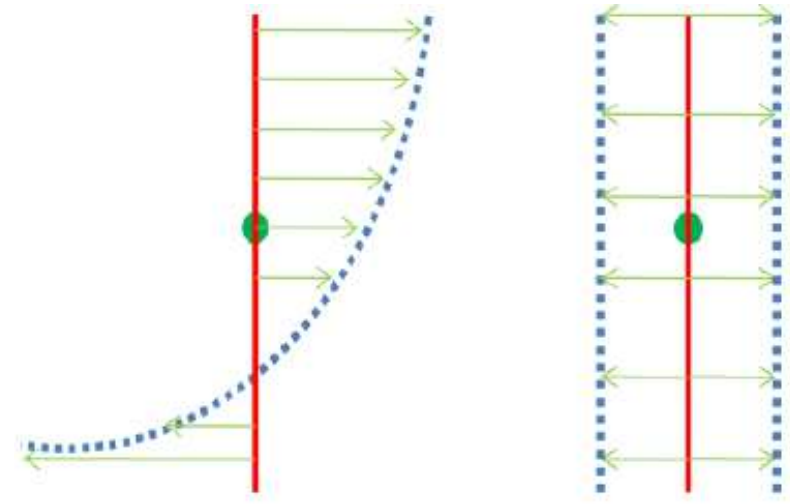

Figure 1: Categorize point clouds of curved structures as roofs (left) or cylinders (right) by examining the vertical distribution of points.

points fine-tuning algorithm were developed to minimize the random and system errors stemmed from the uncertainty in the computation of vanishing points and feature points extractions. The process is illustrated in Fig. 2. Before the correction (Fig. 2a) relative distances from feature points to base points with the same leveling at the left side are longer than right the hand side. The mean and stander deviation for each leveling group after height equalization is displayed in Fig. 2b. Refining the position of vanishing points base on the information in height equalization, more reliable coordinates of vanishing points can be determined and shown in Fig. 2c. Repeating the process can minimize the systematic errors inherited from the uncertainties of vanishing points and random errors to achieve more accurate modeling results.

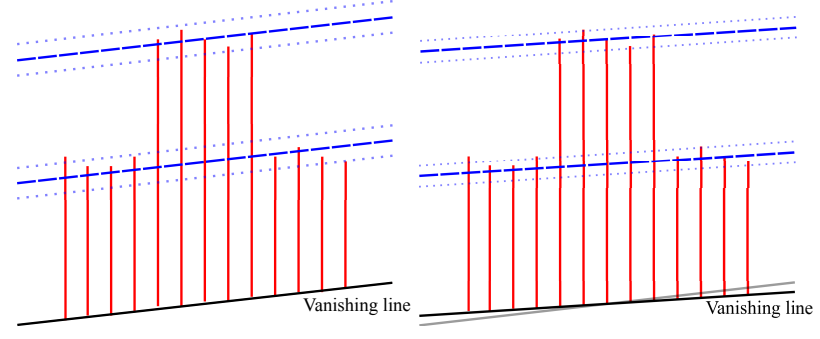

(a) original

(b) height equalization

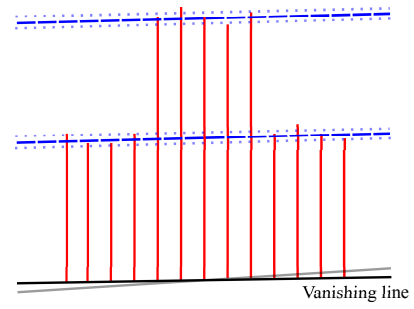

(c) after refinement

Figure 2: Example of height equalization and refinement of vanishing points.

In addition to straight-line features and planar surfaces, a few specific types of curved segments and surfaces can also be reconstructed effectively, including cylindrical structures, surfaces of revolution, and free-form curved surfaces. The principle is similar to the curved surface reconstruction of TLS data. Critical parameters such as center and gravity points, radius, and curve outlines are measured from feature points and the surfaces are reconstructed parametrically or approximated as rule surfaces.

Unlike TLS-based approach, details of building façades are difficult to reconstruct from images directly. However, the level of de- 
tail of the models reconstructed with SVR-based approaches can be increased using rectified façade texture rendered from singleview images. The idea is to treat the SVR results as bare-bone models and perform a semantic analysis of the façade images to identify and model secondary objects, such as windows, doors, balconies and the like (Lee and Tsai, 2010). However, building façade images are often occluded by trees, cars and other street objects. These occlusions can be removed and mended using algorithms described in Tsai and Lin (2007) and Chen and Tsai (2009). This process can not only produce more complete building details but also improve the visual plausibility of the reconstructed models.

\section{EXPERIMENTAL RESULTS}

Figure 3 demonstrates an example of the 3D reconstruction results from LiDAR point clouds. The example shown in Figure is a landmark building (the National Dr. Sun Yat Sen Memorial Hall) in Taipei city, Taiwan. The original data consist of dense point clouds acquired from 8 ground laser scanning stations and a set of sparse ALS roof point cloud. A 3D model of the building was successfully reconstructed from the point clouds using the algorithms described above. In addition to planar façades and structures, non-planar structures, such as the cylindrical pillars and curved roof surfaces were also effectively reconstructed.

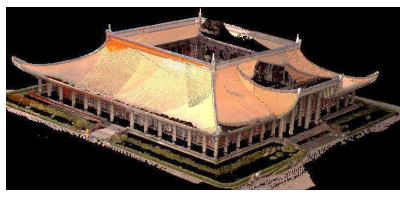

(a) TLS data

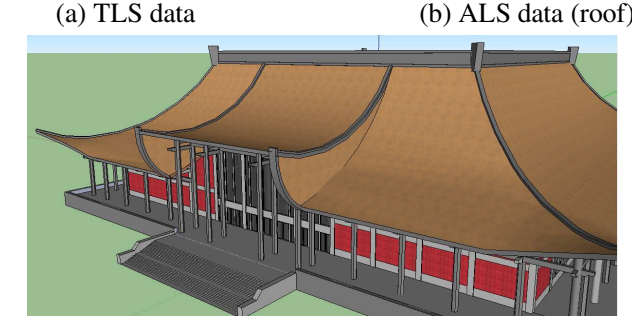

(c) reconstructed 3D model

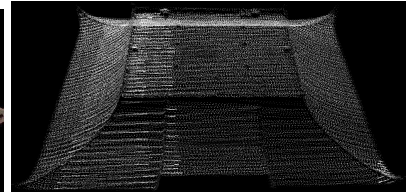

b) ALS data (roof) nstruction of Dr. Sun Yat Sen Memorial Hall Figure 3: 3D reconstruction of Dr. Sun Yat
of Taipei, Taiwan from LiDAR point clouds.

Figure 4 displays the 3D building models of Taipei City Council reconstructed from a single perspective view image. The original image (Fig. 4a) was acquired using a consumer digital camera. After identifying three vanishing points from the detected feature lines in the image, coordinates of detected feature points were successfully obtained and used to create the 3D model (Fig. 4b) conforming to OGC (Open Geospatial Consortium) CityGM LOD2 specification (Gröger et al., 2008). More detailed model (Fig. 4c) can be achieved based on the semantic analysis of the façade images. Quantitative evaluations of the results compared with ground-based surveying indicate that the overall root-meansquare error (RMSE) is less than $2 \%$ for buildings with planar façades, while most of the overall RMSEs for the reconstruction of curved structures can be controlled to be less than $5 \%$.

An advantage of SVR-based 3D building model reconstruction is that it can be applied on old photos or unconventional images such as digitized paintings to reconstruct models of non-existing building buildings, which can be an important task in culture heritage applications. Figure is an example of creating a 3D building model from an acrylics painting. Figure is an old photograph

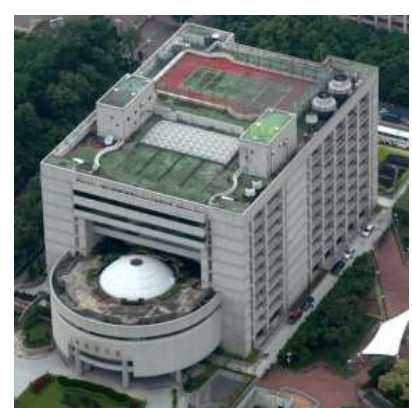

(a) original perspective view image

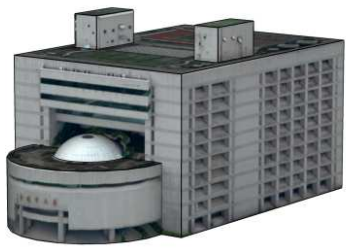

(b) CityGML LOD2 model

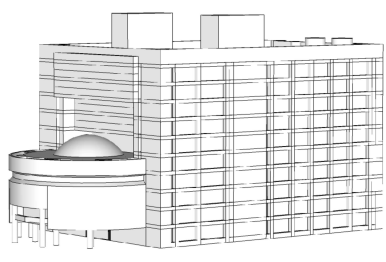

(c) detailed model
Figure 4: 3D reconstruction of a building with non-planar structures from a single image.

showing one of the few remains (the North Gate) of the old Taipei Wall, most of which was destroyed before 1905 by Japanese colonialists. The reconstructed 3D model is played in Fig. b, which reasonably presents the historical appearance of the gate as if in 1950's.
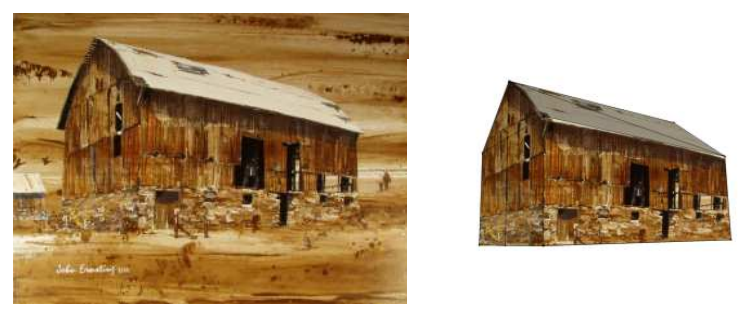

Figure 5: SVR-based 3D building model reconstruction from an acrylics painting.

\section{CONCLUSIONS}

The TLS procedure demonstrated in this paper can successfully create detailed 3D building models from LiDAR point clouds acquired with ground-based laser scanners; while the SVR approach can reconstruct 3D building models from single-view perspective imagery, including unconventional images. In addition to planar façades, curved surfaces can also be reconstructed or approximated in both approaches. Examples presented in this paper also demonstrate that both approaches can generate detailed models of landmark or historical buildings. The SVR-based 3D reconstruction can even be utilized to reconstruct models of nonexisting buildings from old photographs or unconventional images. These can be considered feasible methods for digital documentation or archiving of buildings in terms of culture heritage applications.

\section{ACKNOWLEDGEMENTS}

This study was partially supported by the Ministry of Interior of Taiwan under project No. SYC1030127. 


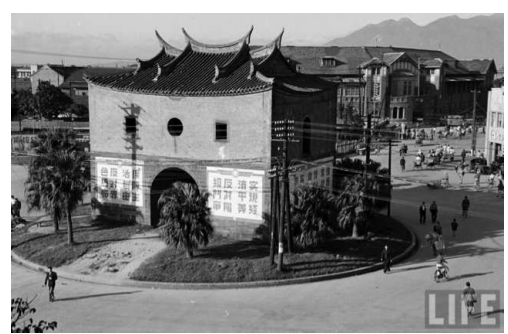

(a) original photo taken in 1950 by Carl Mydans (CTime inc.
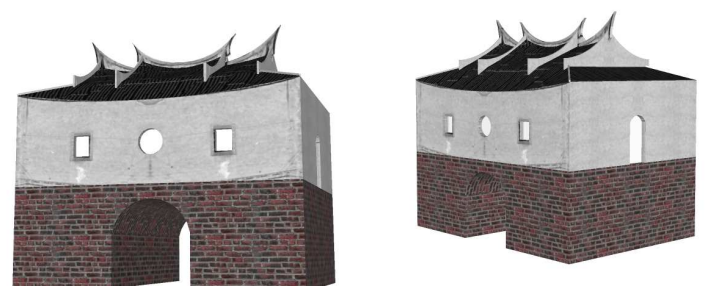

(b) reconstructed model with synthetic texture

Figure 6: 3D reconstruction of Taipei North Gate from an old photograph.

\section{References}

Chang, H. and Tsai, F., 2009. 3D building model reconstruction from a single image. Asian Journal of Geoinformatics 9(4), pp. 41-44.

Chen, P.-S. and Tsai, F., 2009. Occlusion removal of facade texture with constrained image inpainting. In: Proc. 30th Asian Conference on Remote Sensing, Beijing, China.
Criminisi, A., Reid, I. and Zisserman, A., 2000. Single view metrology. International Journal of Computer Vision 40(2), pp. $123-148$.

Fischler, M. A. and Bolles, R. C., 1981. Random sample consensus: A paradigm for model fitting with application to image analysis and automated cartography. Communications of the ACM 24(6), pp. 381-395.

Gröger, G., Kolbe, T. H., Czerwinski, A. and Nagel, C., 2008. OpenGIS city geography markup language (CityGML) encoding standard. Technical Report OGC-08-007r1 v1.0.0, Open Geospatial Consortium Inc.

Lee, M.-J. and Tsai, F., 2010. Increase the level of detail of 3D building models with close-range images. In: Proc. of 31st Asian Conference on Remote Sensing (ACRS2010), Hanoi, Vietnam.

Pu, S. and Vosselman, G., 2009a. Building facade reconstruction by fusing terrestrial laser points and images. Sensors 9(6), pp. $4525-4542$.

$\mathrm{Pu}, \mathrm{S}$. and Vosselman, G., 2009b. Knowledge based reconstruction of building models from terrestrial laser scanning data. ISPRS Journal of Photogrammetry and Remote Sensing 64(6), pp. $575-584$.

Tsai, F. and Lin, H.-C., 2007. Polygon-based texture mapping for cyber city 3D building models. nternational Journal of Geographical Information Science 21(9), pp. 965-981.

Wang, G., Hu, Z., Wu, F. and Tsui, H. T., 2005. Single view metrology from scene constraints. Image and Vision Computing 23, pp. 831-840. 